\title{
asasim: Adaptive sampling for electromagnetic simulations
}

\section{Sørensen, Kristian Tølbøl}

\section{Published in:}

Computer Physics Communications

Link to article, DOI:

10.1016/j.cpc.2018.10.017

Publication date:

2019

Document Version

Peer reviewed version

Link back to DTU Orbit

Citation (APA):

Sørensen, K. T. (2019). asasim: Adaptive sampling for electromagnetic simulations. Computer Physics Communications, 236, 268-273. https://doi.org/10.1016/j.cpc.2018.10.017

\section{General rights}

Copyright and moral rights for the publications made accessible in the public portal are retained by the authors and/or other copyright owners and it is a condition of accessing publications that users recognise and abide by the legal requirements associated with these rights.

- Users may download and print one copy of any publication from the public portal for the purpose of private study or research.

- You may not further distribute the material or use it for any profit-making activity or commercial gain

- You may freely distribute the URL identifying the publication in the public portal

If you believe that this document breaches copyright please contact us providing details, and we will remove access to the work immediately and investigate your claim 


\section{Accepted Manuscript}

asasim: Adaptive sampling for electromagnetic simulations

Kristian Tølbøl Sørensen

PII:

S0010-4655(18)30365-5

DOI:

https://doi.org/10.1016/j.cpc.2018.10.017

Reference: COMPHY 6643

To appear in: Computer Physics Communications

Received date: 5 November 2017

Revised date: 10 October 2018

Accepted date : 14 October 2018

Please cite this article as: K.T. Sørensen, asas im: Adaptive sampling for electromagnetic simulations, Computer Physics Communications (2018), https://doi.org/10.1016/j.cpc.2018.10.017

This is a PDF file of an unedited manuscript that has been accepted for publication. As a service to our customers we are providing this early version of the manuscript. The manuscript will undergo copyediting, typesetting, and review of the resulting proof before it is published in its final form. Please note that during the production process errors may be discovered which could affect the content, and all legal disclaimers that apply to the journal pertain. 


\title{
asasim: Adaptive Sampling for Electromagnetic Sını ’lations
}

\author{
Kristian Tølbøl Sørensen ${ }^{\mathrm{a}, *}$ \\ ${ }^{a}$ DTU Nanotech, Technical University of Denmark, Ørsteds Plads building .'5C, $2800 \mathrm{Kgs}$. \\ Lyngby, Denmark.
}

\begin{abstract}
For simulations of electromagnetic resonance spectra $\left.\mathrm{w}^{\mathrm{l}}\right\lrcorner \mathrm{re}$, he locations of spectral features are unknown, and for wide-band simulatior. in gen ial, a substantial number of wavelengths must be simulated for acceptable resolu i on, increasing computation time. This problem is exacerbated for spectra con inin, narrow-band features, as a

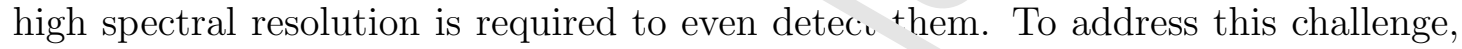
a heuristic algorithm is presented for electrc agneuc simulations, which adaptively refines the local resolution of spectral features "'ring a simulation. The method supports parallel processing and plugs in it tristing simulation systems, such as rigorous coupled-wave analysis (RCWA' It, ?n routinely reduce the computational load by two orders of magnitude.
\end{abstract}

Keywords: lorentzian; adaptive; resu'-1tıun, rcwa; PACS 40; PACS 41

\section{PROGRAM SUMMARY}

Program Title: asasim

Program Files doi: http://d .do . or $/ 10.17632 /$ d6gty $7 \mathrm{kr} 2 \mathrm{x} .1$

Licensing provisions: CC By ' $\mathrm{C}$

Programming language: $\mathrm{M}+\mathrm{TLAL}$

Nature of problem: Sim lat. 'ns are challenging when information is needed both on a

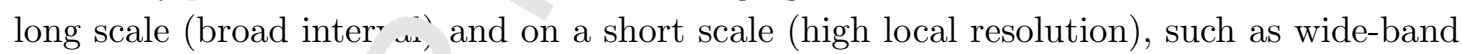
electromagnetic $\operatorname{spec}^{+}$a c ntaining narrow-band features. When resolution is insufficient, narrow-band featur is $m_{a}$, be downright absent from the spectrum, if neighboring points are simulated on ith $r$ sire of a narrow peak. When local resolution is sufficient, it will necessarily be exces re ; 1 flat regions, wastefully increasing computation time.

Solution meth $d$ : Tl o presented method enables adaptive resolution, which ensures that

\footnotetext{
${ }^{*}$ Corres ,ondin * author.

E-mail ad.ress: kr stian@tolbol.dk
} 
all peaks of a given minimum width are always detected and maximally resu red, while feature-less regions remain minimally resolved. An optimum point spe 11, $_{\text {, is derived for }}$ lorentzian peaks (descriptive of optical resonances) and is applied to or -im; $\lrcorner$ e computation time. 


\section{Introduction}

The ability to accurately simulate light-matter interac tions is nanostructures has enabled breakthroughs in areas as diverse as optical bi census [1], pigment-free coloration [2], and solar cells [3]. Rigorous coupled-wav an wy: s (RCWA) is a popular semi-analytical method for electromagnetic simula ${ }^{i}$ ons ori snally described by Moharam and Gaylord in 1981 [4]. However, the methou is computationally demanding, and this can be a limitation for high-resolu 'nn, $\mathrm{v}$ de-band simulations. This can be particularly problematic for optimizatio. metnods, such as particle swarm optimization [5] or genetic algorithms [6], $\mathrm{w}^{1} \ldots \ldots$.... xtensive number of simulations in a many-dimensional parameter space should $s$ - performed. Such endeavors would benefit from increased simulation efficienc.

The challenges of multiscale simulations, tve similarly been encountered in other fields, where more intricate schemes have $u\urcorner n$ demonstrated, e.g., for elastodynamic shock propagation[7] or particle-parislo in ${ }^{+}$eractions[8].

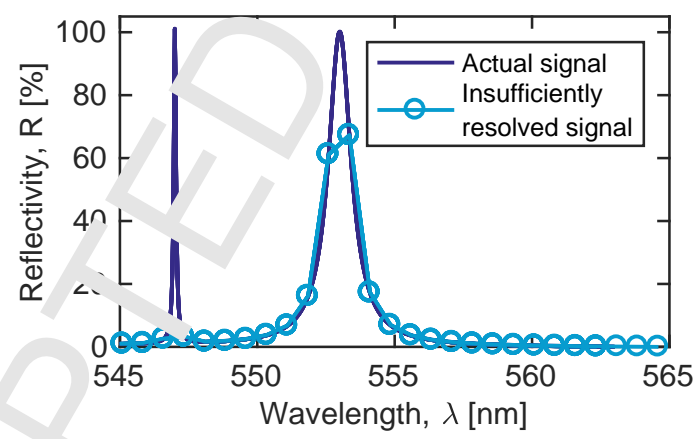

Figure 1: Illustrati $\mathrm{n}$ of detection problem. At insufficient resolution, the peak at $547 \mathrm{~nm}$ is not registered at all, as s neir aboring points may randomly fall on either side of it. The much wider peak at $553 \mathrm{~nm}$, ertalı o be detected at this resolution, but it is still poorly resolved.

Simulation time can be reduced by simply reducing the spectral resolution. However, wh $\mathrm{n}$ sim lation resolution becomes too low, narrow peaks may not even be detected, as illu trated in figure 1. Depending on whether a data point happens to 
fall on the narrow peak, it may or may not register as a bump, but th $\bullet$ will be a risk that the peak is completely absent from the simulation, $\mathrm{w}^{\mathrm{t}} \boldsymbol{\omega}$ can be quite misleading.

As also illustrated in figure 1 , peaks of sufficient width wili ' ${ }^{\prime}{ }_{1} \iota_{1}$, be detected, as at least one point will always fall on the peak. However, "esor +ion may still be too low to properly resolve its lineshape, which is comm onl a . imulation goal. An obvious solution is to increase the spectral resolution of $t_{\iota_{1}}$ simulation, proportionally increasing simulation time, but this would resu' $v$ in $~ ₹ n$ unnecessarily high resolution in the flat parts of the spectrum. Figure 2A is 'ustrat s this central issue, i.e., spectrally flat regions are over-emphasized, whe ws regions with features are under-emphasized. Ideally, an initial simulation shor ld ,nly be fine-grained enough that the presence of a peak would always be detect $r$, ar $r$ seak regions should then be further resolved to the desired resolution.

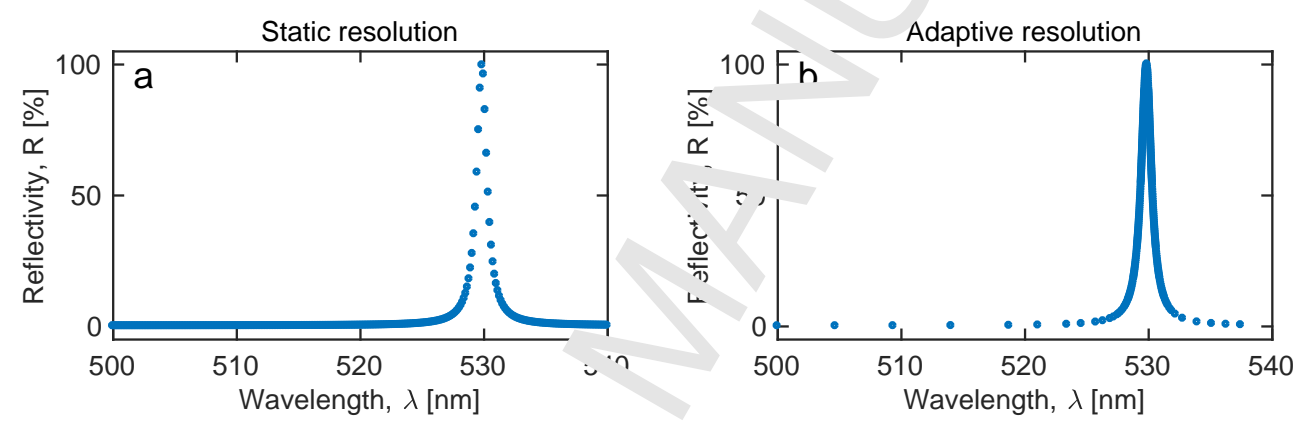

Figure 2: The same peak simulated with . 79 points using a) static resolution, and b) adaptive resolution. The static method yieic a higl information density off-peak, whereas the adaptive method emphasizes the peak regi n.

Here, a heuristic Mf $\mathrm{MLA}$. $\mathrm{g}$ gorithm is demonstrated for achieving adaptive resolution in electroma', $\mathrm{m}^{\text {+ic }}$ simulations. This effectively reverses the information emphasis to lay on the spectral features rather than the background, as illustrated in figure 2b. Furth rms ce, optimal parameters are derived, and the speed of the method is evaluated.

\section{Examples an' in ' callation}

At its co e, the isasim algorithm simply replaces the per-wavelength for-loop of a typical en nagnetic simulation system. Thus, instead of statically looping over all v tveler rths in an interval with identical spacing, as is the common approach, asasim e qluate, which regions to refine during runtime, thereby only refining regions 
where features are present. Therefore, in principle, any script that cals a : mulation function from a for-loop can be integrated with asasim. The syst 11 verforms best for "needle-in-a-haystack"-type simulations, where narrow-band sat res are found in a broad interval. As this is common for electromagnetic reso. nce nectra, these are the focus of this paper, although the system will likely ${ }^{\prime}$ so $\mathrm{m}$ applicable to many other topics of simulation. This section explains ho $v$ a as: $n$ is integrated with existing simulation systems in MATLAB 2016b, runniı, on a MacBook Pro (2.4 GHz Intel Core i5, 8 GB $1600 \mathrm{MHz}$ DDR3 RAM).

\subsection{A simple test}
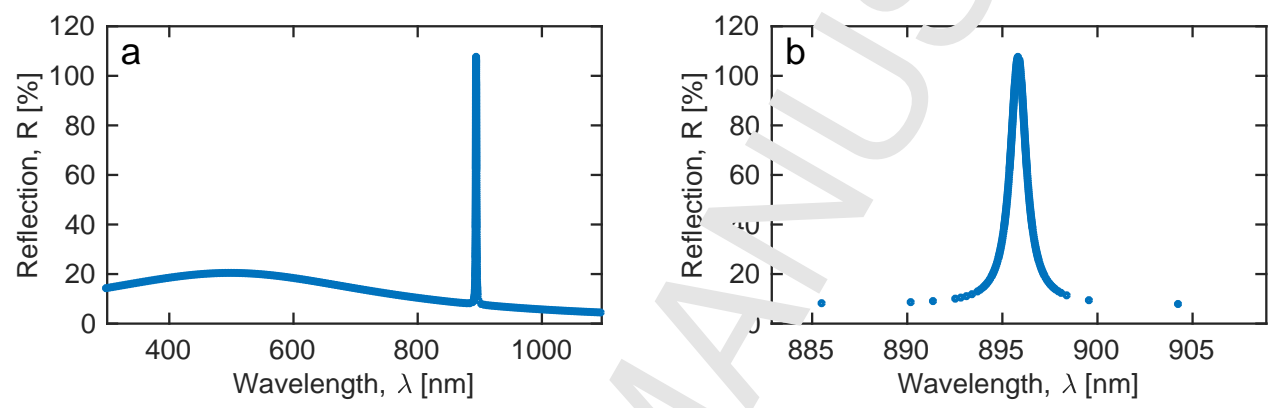

Figure 3: Output of example script, whi ' : mitia os an electromagnetic simulation at a random position a) within a wide interval, with b) na now line width. The resolution varies from $4.6 \mathrm{pm}$ on the peak, to $4.6 \mathrm{~nm}$ in the flat parts of the spectrum. Achieving the same resolution with static spacing would require $370 \times$ as many som

As actual electromagnetir simu ${ }^{*}$ : ons can be complex to set up and time-consuming to run, a test-function was wri' ien that imitates an actual simulation function, but returns values from an ar aly $, a, l$ svaluation of lorentzians. The script

asasim_Example_1_Imi+a ${ }^{\prime} n r . m$ exemplifies how the asasim method may be integrated with an arbitrary sin lation function. A lorentzian of narrow line width $(0.5 \mathrm{~nm})$ is simulate at a random location within the broad interval $300-1100 \mathrm{~nm}$, on a slightly sloped - kground, given by a broad lorentzian. The time to run is $\sim 1$ second, and f surr 3 suows the results.

\subsection{Photonic nnystu' ab sensor at varying angles of incidence}

GD-Calc is a A ATLAB-package for RCWA, which can be downloaded from

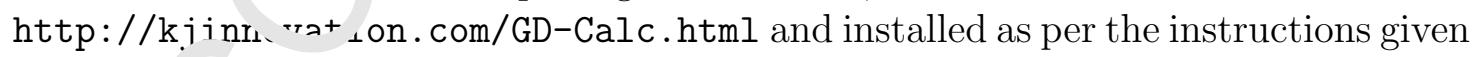
on the $\mathrm{w}$ osite. The workhorse of the GD-Calc package is the function gdc.m, which takes thre inpu s: a Grating-struct with the relevant optical grating parameters and 


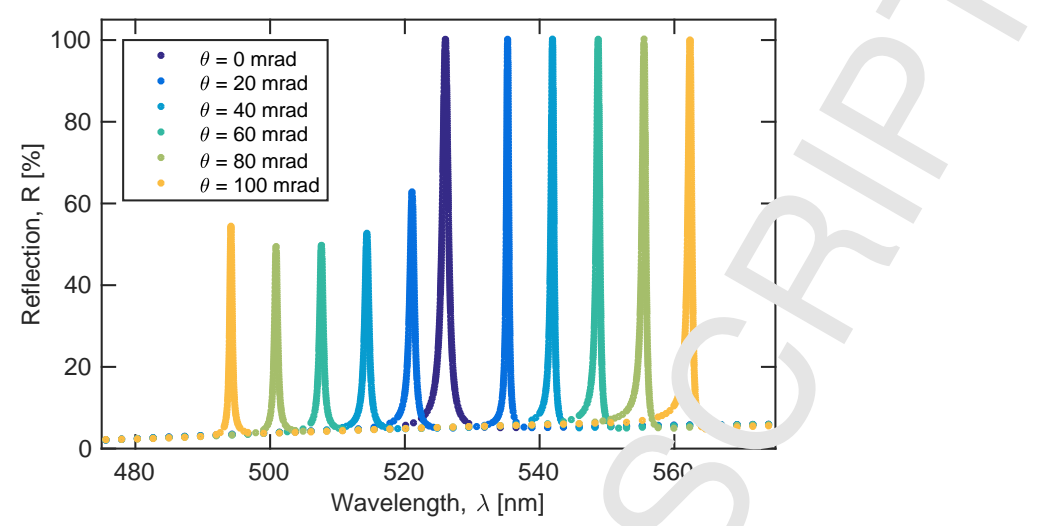

Figure 4: Example simulation of PCS sensor at varying angles ` ' incidence. Each simulation has a variable resolution between $2 \mathrm{pm}$ and $2342 \mathrm{pm}$ dependir. on the scal region.

geometric layout, an IncField-struct with in ${ }^{r}$........ivivn about the incoming field, and an order-matrix, specifying the diffraction orde ${ }_{1}$ to be used in the calculations. The function gdcWrapper has been written to . רe, ... a asasim with GD-Calc. It defines these necessary three inputs and calls gdc.m o get a reflectivity value $R$ at the given input wavelength w. An additional Fui.' $u^{+}{ }^{+}$but struct contains all diffraction efficiencies. asasim uses this reflectivit *alue $q$ to evaluate whether points neighboring w should be further resolved. The asas: $m$ system was recently used with RCWA to substantiate experimental observations of waveguide core swelling [9] in a photonic crystal slab (PCS) sensor. Her, the s mulation parameters describe a linear grating of period $368 \mathrm{~nm}$, duty cycle $50 \%$ nd srating height $100 \mathrm{~nm}$, illuminated at an angle $\theta$. The model incorporates refractive index dispersion data for all three materials constituting the sensor, na. ol/a adding layer of Efiron PC409AP (Luvantix, Korea), a nano-structured ore lay - of HI01XP (micro resist technologies, Germany) and water as superstra e. 1. the example given here, simulations are performed in a broad wavelength int . . . l of 450-850 nm, with angles of incidence in steps of $0.02 \mathrm{rad}$ between 0 and $0.1 \mathrm{r} \cdot \mathrm{d}$. ' ' 'he full example code is given in asasim_Example_2_GDC.m, and the result is shown $\eta$ figure 4 . It should be noted that asasim is incapable of displaying a pre res, bar during runtime due to parallel processing working asynchronously, and be $\neg \mathrm{u}$ ( 2 the wavelengths to simulate are being refined adaptively, and are thus not $\mathrm{k}$ own a priori. Instead, the total number of points calculated is displayed for ach $r$ ' und to indicate progress. On the computer used here, the total run time $f_{J 1}$ all $u$ angles is $\sim 10 \mathrm{~min}$. 


\subsection{Scattering cross section of plasmonic nanoparticles}
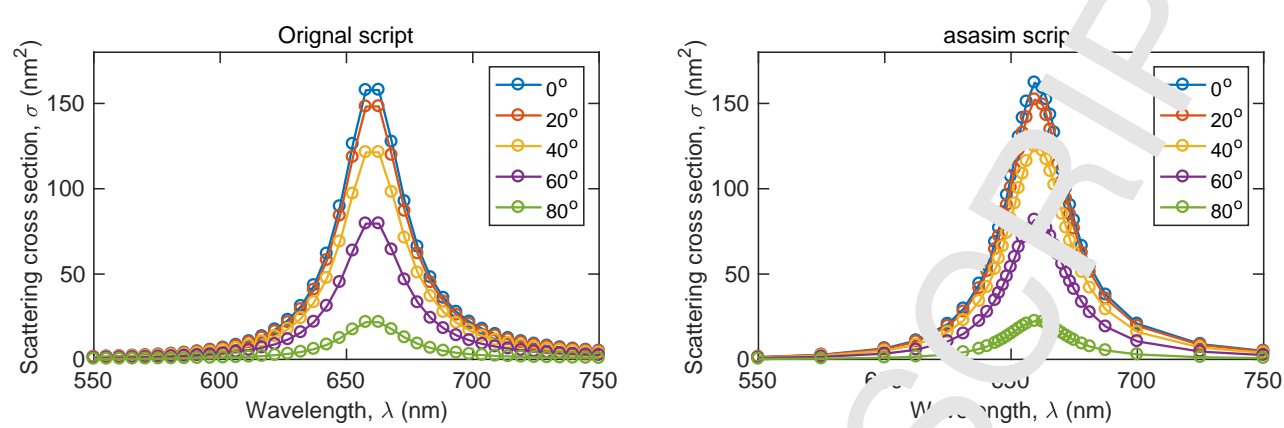

Figure 5: Simulation performed using the mnpbem-package. a, 'Tsins ${ }^{2}$ 'e default static spacing places 200 points, many of them off-peak. b) With adaptive resolution, $\mathrm{c}$. e peaks are better resolved using only 165 points.

To demonstrate the versatility of asasim it m... so integrated with the excellent class mnpbem[10], available at http://cpc.cs.q. h.ac.uk/summaries/AEKJ_v3_0.html, which is used for calculating scattering crc s ${ }^{-i n n s}$ of plasmonic nanoparticles near surfaces. An example script, based the demo' tration demospecstat1.m of that toolbox, is given in asasim_Example_3_MNP... ' ' Jer., the per-wavelength for-loop is quite simply replaced by a call to asasim using mnpWrapper, which is functionally three lines long. As mentioned in section 2.4, the wrapper must take two inputs and return two outputs, and it serves as an example of how simply a wrapper function can be written. The resulting graph ; sho $n$ in figure 5, and although there is perhaps limited gain from using asasin ' $\urcorner$ ' sir ulating such wide peaks in a limited interval, the example illustrates how idditionsl simulation systems can be interfaced using a wrapper function. The coc $\mathrm{h}$ s a ypical runtime of $\sim 3$ seconds.

\subsection{Installation}

The contents of as _ im.zip should be decompressed to a folder on the MATLAB search path, such ar MAT $\angle A B / T o o l b o x e s / a s a s i m$. With the MATLAB-folder as the working directory, scrip should always contain the command addpath (genpath (pwd)) in order to add : 1 f fi' es in all subfolders to the search path. This line is included in all example files.

The mair system for adaptive resolution is now installed, and is ready to be interfaced wi $\mathrm{h}$ an e isting simulation system. In general, conversion of a simulation script (list: $-{ }_{-}$1) inwo a version utilizing adaptive resolution (listing 2), is a three-step operatiol : 
Listing 1: Original code

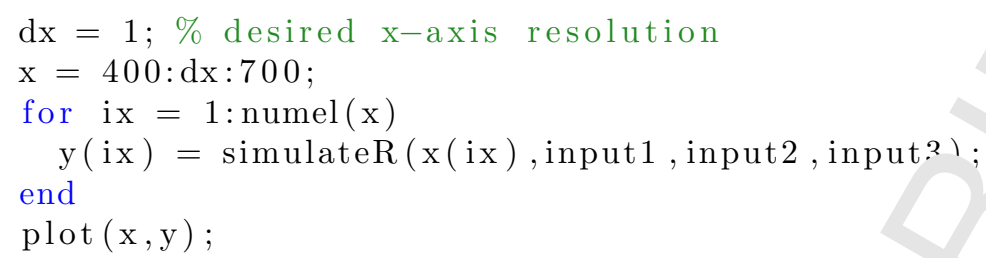

Listing 2: asasim-implementation, illustrating how a wrappe -functic 2 may be defined.

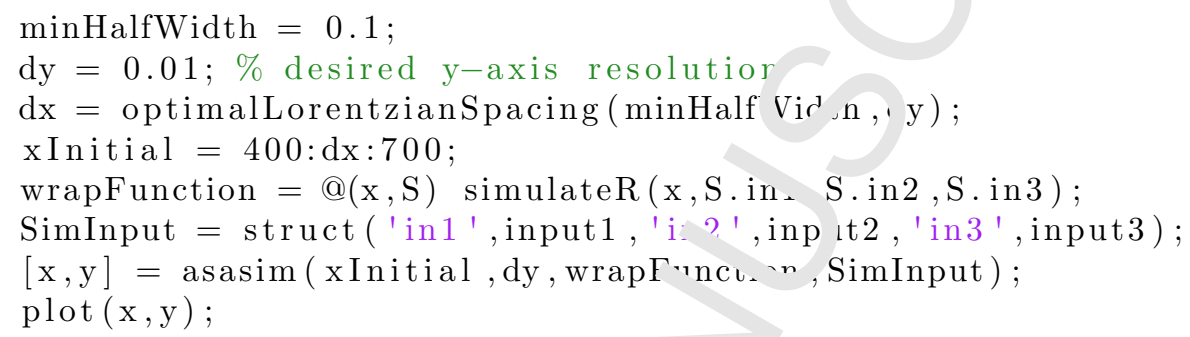

1. Define wrapper. Copy the conten of vis per-wavelength for-loop into a wrapper function.

2. Determine initial point spacing. To neaks described as lorentzians ranging from 0 to $100 \%$ intensity, the r $^{+i m}, 1$ point spacing can be calculated automatically using the optimalLoren $>$ ianSpacing function.

3. Replace the per-wavelengtr ${ }^{\wedge} \cap r$-loop by a call to asasim.

Each of these steps will be ela'nrate. next in the context of concrete application examples.

The purpose of the wr op $\mathrm{r}$ f nction is to bridge the simulation system with asasim, and must take to $\mathrm{in}_{\mathrm{r}}{ }^{+}$, , namely the simulation coordinate $\mathrm{x}$ (e.g., wavelength) and a struct of ar 'itional parameters. The wrapper function passes these parameters on to the cimulatiun function(s), using the specific syntax of that simulation system. In gr ner; 1 , the content of a per-wavelength for-loop can often just be excised and plarea: a wrapper function, and the for-loop itself is then replaced by a call to asa im This was first exemplified in the simple case above, where only a single hyp ${ }^{+}$'etir al function simulateR was called from within the for-loop. Subsequent e anples vemonstrated how more involved cases could be handled.

For simu ations vhere the peak-shape resembles a lorentzian with a maximum intensity of $1 儿, 7$, ne optimum initial point spacing $d x$ can be calculated by calling optimal oren ianSpacing, where minHalfWidth is the minimum lorentzian halfwidth to 'e de'ected, and yRes is the desired y-axis resolution. Using this point 
spacing, a vector of points to be calculated initially is then definc $d$ as - Tnitial $=\mathrm{x} 1: \mathrm{dx}: \mathrm{x} 2$, where $\mathrm{x} 1$ and $\mathrm{x} 2$ represent the extremes of the intf $\mathrm{ve}^{\prime}$ to simulate. Alternatively, if the spectrum does not contain lorentzian peaks a $10, \%$ maximum, arbitrary values for dy and xInitial can be defined manually, u ing a 'ustom point spacing $\mathrm{dx}$. The following section explains how and why an or' 'nal $r$ 'int spacing is found.

\section{Background}

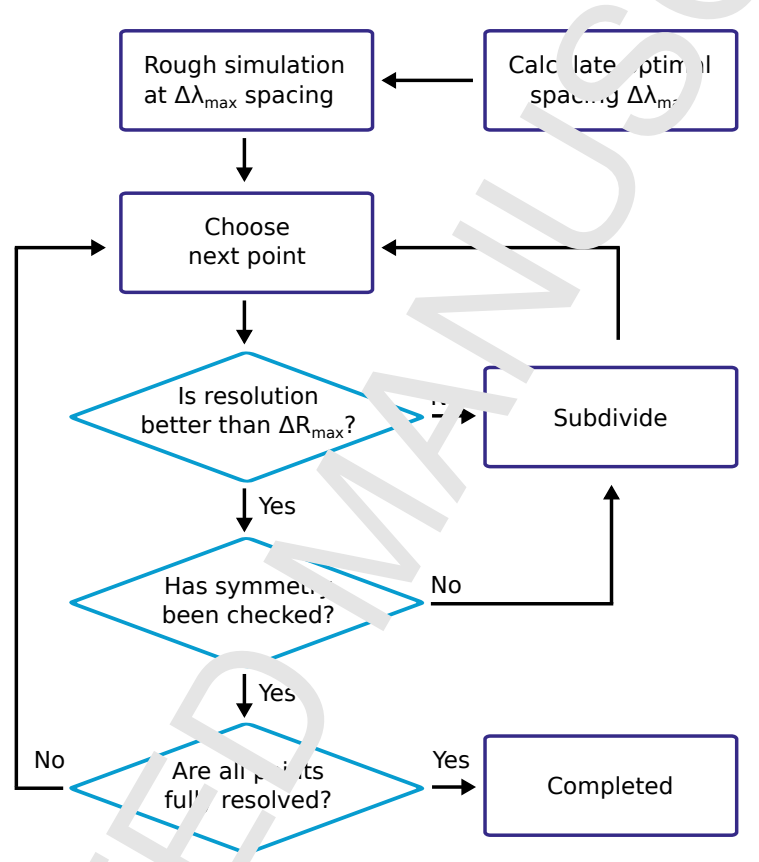

Figure 6: Working princip' ' shind the algorithm. An optimal point spacing is calculated using equation 5, and an initial rouc 1 simulation is performed in order to detect all relevant features. Then, each peak ; th ther resolved by adaptive subdivisioning until the desired resolution is achieved. At this po ' $\mathrm{t}$, a symmetry-check is performed in order to ensure that two points with similar values are not just ${ }_{\vdash}{ }^{\prime}$.ced symmetrically around a peak.

A simplified $11 \cdot \cdot$ tra $^{\prime}$ on of the working principle is presented in figure 6 , with the goal of prodv $\operatorname{lng}$ a $(\wedge, R)$-spectrum using $\Delta R_{\max }$ (goal resolution) and $\gamma_{\min }$ (minimum peak h. (f-widt l) as input parameters. First, an optimum initial point spacing $\Delta \lambda_{\max }$ is $\cdots$ toln $_{\text {. }}$ ically calculated, such that any peak of a given minimum halfwidth $\gamma_{\mathrm{n}}$ is ct tain to cause a perturbation exceeding the threshold $\Delta R_{\max }$ within 
the interval, flagging the region for further refinement. Then, a rous $\mathrm{n}$ s1.. lation is performed, with the purpose of detecting all spectral features of in es st.

After each round of simulations, the difference $\Delta R$ between - oig' 'boring points is evaluated, to identify regions that exceed $\Delta R_{\max }$. These will $i$ ' fur ${ }^{\prime} \circ$ or refined by subdivisioning. New points inherit the $\Delta R$-value of its parer' in t t. ${ }^{+}$round, such that subdivisioning does not continue indefinitely.

When a point no longer exceeds $\Delta R_{\max }$, it is subdivide one last time as a symmetry-check. It is entirely possible for two points $t\lrcorner$ be ilaced symmetrically around a peak, in which case their difference $\Delta R$ could ve zerc, without the upper part of the peak having been resolved. The symmr uy-cricuk safe-guards against this. If the difference still does not exceed $\Delta R_{\max }$, th $-\mathrm{r} \in$ rion is considered fully resolved. Thus, once a peak is detected, i.e., at lea ${ }^{2}$ one $n$, int satisfies the criterion $\Delta R>\Delta R_{\max }$, the entire peak always becomes fully res. lved.

For the sake of argument, consider a lorent's $n a^{\dagger}$ an arbitrary location, i.e., $\lambda_{0}=0$, normalized so that $R\left(\lambda_{0}\right)=100 \%$ :

$$
\begin{aligned}
R(\lambda) & =\frac{1}{1-\gamma^{2}} \\
\lambda & =V^{\gamma}\left(\frac{1}{R}-1\right)
\end{aligned}
$$

Because of the subdivisioning-schen employed, whenever a peak is detected, it is certain to come out fully $\mathrm{r}^{r^{-1}}$ ved. Detection in this context entails that the perturbation from a peak cav es twc neighboring points, spaced apart by $\Delta \lambda$ on the first axis, to have a suffirien diff rence on the second axis, $\Delta R>\Delta R_{\max }$. The narrowest peak of half-widt $\lrcorner \gamma$ hat is certain to be detected is then a peak that is so narrow, that its perturba: in $\mathrm{c}$.ly just causes $\Delta R$ between any two neighboring points to exceed $\Delta R_{\mathrm{me}}$. even when the peak is placed right between those two neighboring points, such as nints $a$ and $b$ in figure 7 . In this case, $\Delta R$ is zero between them, so for at ection, the difference to the next neighbor (point $c$ ) must instead satisfy $\Delta R>\Delta R_{\max }$. If the distance from the peak center to the first symmetrically ple ed ne $e_{c}$ hbor (point $b$ ) is $\lambda_{1}=\Delta \lambda / 2$, then the distance to its next neighbor (point · r ust je $3 \lambda_{1}$. Thus,

$$
\begin{aligned}
\Delta R & =R_{1}-R_{2} \\
& =\frac{1}{1+\frac{\lambda_{1}^{2}}{\gamma^{2}}}-\frac{1}{1+\frac{\left(3 \lambda_{1}\right)^{2}}{\gamma^{2}}}
\end{aligned}
$$


This does not have a simple solution for the optimal point spacing $\angle \lambda=2 \mathrm{I}_{1}$, but it can be isolated as

$$
\Delta \lambda=\left(\frac{2}{3} \sqrt{\frac{\gamma^{2}(4-5 \Delta R)+2 \sqrt{2} \sqrt{\gamma^{4}\left(2 \Delta R^{2}-5 \Delta K\right.}}{\Delta R}}\right)
$$

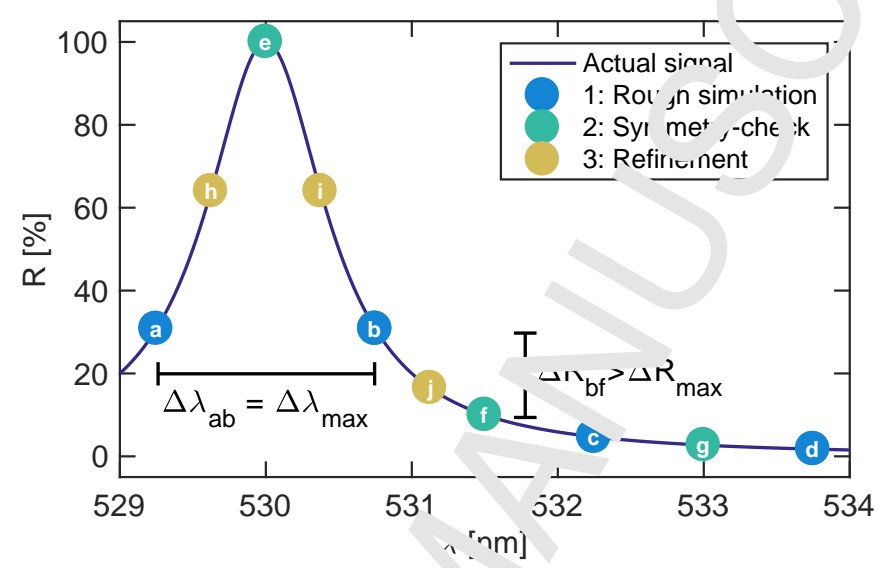

Figure 7: Illustration of points placed in rough-simulation is performed in order to det + all relevant features. e-g) A symmetry-check is performed in order to ensure that two noints with similar values are not just placed symmetrically around the peak (like points a and $\mathrm{k}, \mathrm{h}-\mathrm{J}$, Deak refinement continues until a desired resolution is achieved. Note that here, the spect. ' $\mathrm{m}$ beyr nd $\lambda>531.3 \mathrm{~nm}$ is therefore not refined after round two.

\section{Discussion}

The optimum rati, ' hetween the parameters $\Delta \lambda_{\max }$ and $\Delta R_{\max }$ for a given $\gamma_{\min }$ is given by equatior 5 , nd shown in figure 8 . Parameter-sets below the curve are sub-optimal in th sen that they cause more simulation points to be calculated than necessary, rast fully increasing computation time. Sets above the curve will only serendipitous, re olve peaks of a given half-width $\gamma_{\min }$. As an example, if a simulation is 0 be nerformed with a $5 \%$ resolution on the y-axis, and the expected minimum ha ${ }^{\circ}$-widtl $\gamma_{\min }$ is $1 \mathrm{~nm}$, the optimum spacing on the $\mathrm{x}$-axis is $8.2 \mathrm{~nm}$. At a resolution $0.5 \%$, the spacing can be $26.6 \mathrm{~nm}$, while still detecting the perturbation from the narron peak. 


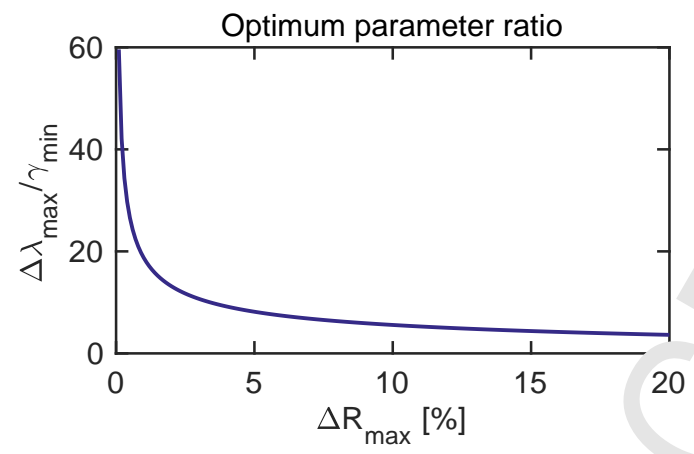

Figure 8: Optimum ratio of simulatios pa ${ }^{\star}$ mt ers.

Decreasing $\Delta R_{\max }$ causes more points to be calcula ed on the $R$-axis, but this also increases $\Delta \lambda_{\max }$, reducing the number of prinu to se calculated initially on the $\lambda$-axis. In the simplest possible model, consider a nectrum only containing a single lorentzian peak with a half-width of $\gamma$. The. . "mber of points simulated on the fully resolved peak is $N_{R}=2 / \Delta R$, and the nimber un points to be simulated statically across the spectrum is $N_{\lambda}=\left(\lambda_{\max }-\lambda_{\min }, / \Lambda \cdot \lambda\right.$. The total number of points to be simulated adaptively is

$$
N=\frac{\cap}{\Delta R_{\max }}-\frac{\lambda_{\max }-\lambda_{\min }}{\Delta \lambda_{\max }}
$$

Using this equation for estimati $\mathrm{g}$ tı number of simulation points, the two methods were compared for speed as sh wn in igure 9. It is clear that the adaptive method is generally a couple of ord ars of 1 - agnitude faster than the static method. The figure also illustrates the $\mathrm{c} m p$ tat onal optimum (curve minimum) for the $\Delta R$ and thus $\Delta \lambda$ parameters, whi $\mathrm{n} \mathrm{de}_{\mathrm{r}}{ }^{\mathrm{r}} \mathrm{d}$ on the minimum necessary peak half-width $\gamma_{\min }$. Time-optimal parametf s u uld be determined by combination of equations 5 and 6 and solving $\frac{d N}{d \Delta R_{\max }}=n$ but tris becomes rather unwieldy. As figure 9 indicates, the total number of sim tate $\mathbb{l}$ points does not vary steeply for similar values of $\Delta R$, and so the choice of resolu n is perhaps more a question of preference.

For comparis $\mathrm{n}, i_{1}$ order to achieve the same $R$-axis resolution with static sampling as with aac ${ }^{\prime}$ ive ampling, the static first-axis point spacing must equal the smallest distr . .ce between two points separated by $\Delta R_{\max }$ on the second axis. For example, to ssolve $\iota$ peak of half-width $\gamma=0.5 \mathrm{~nm}$ at $\Delta R=1 \%$ resolution on the steepest part, $1 . \cdots 800 \mathrm{~nm}$ interval, the adaptive resolution varies between 0.0046 $\mathrm{nm}$ and t.6 $\mathrm{nm}$. depending on the local spectral features. To achieve a static resolution $0_{1} \cap .004, \mathrm{~nm}$, more than 170,000 points would be required. With adaptive 
resolution, the same is achieved with 468 points, making the simulation $3 i^{\prime} \times$ faster.

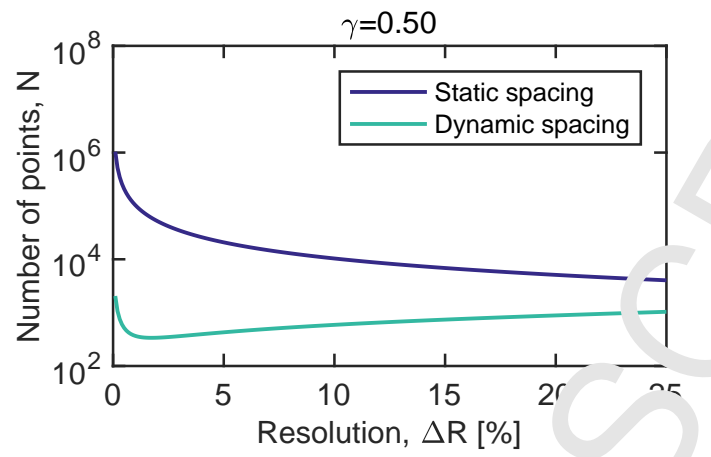

Figure 9: Number of points simulated as function $f R$-axis resolution.

Apart from reducing computation time, a $\mathrm{m}_{1}$-in auvantage of asasim is the decreased amount of a priori information neernd 1 - pectrally flat regions are com-

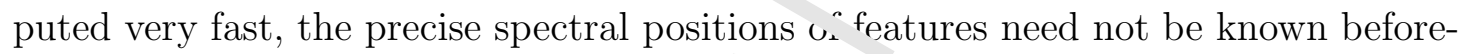
hand in order to simulate the narrow regi $4 \div$-nlevance. Furthermore, whereas one might have to iteratively adjust first-axis $r_{t}$ slution in order to achieve the desired second-axis resolution, with this metho ', resulution is decided for the second-axis directly.

While the amount of necessary $a p^{\prime}$ ' $r i$ information is reduced, a rough estimate of the smallest realistic half-width is still required. The consequences of choosing a poor value for this parameter $\mathrm{w} s$ disc issed in section 4 . For very dense spectra, e.g., containing many closely spacea ${ }_{\uparrow}$ 'aks ' uch as interference patterns, the same amount of points may end up being s mulateu as using static spacing. Furthermore, when the background is strongly slor - $d$. e.g. the resonance peak of interest resembles a bump on a larger and much br sader, ak, the background also becomes highly resolved. This is partially the ca' 2 1. figure 5 .

Because the x-axis - solution varies across all spectra, direct comparison between spectra or presentat on f data as an image will require interpolation. This is quite simply achieved us:ng $\iota_{\text {. }}$ o built-in MATLAB-function interp1, as exemplified below.

\section{Summary}

In summi ry, the presented method allows high-speed, high-resolution simulation of narrow-honu . . tral features in a broad range, with no a priori information about the locat on of pectral features. In one example, the number of points necessary to simula ? was reduced from $>170,000$ to 468 , with an accompanying reduction in 
Listing 3: Interpolation

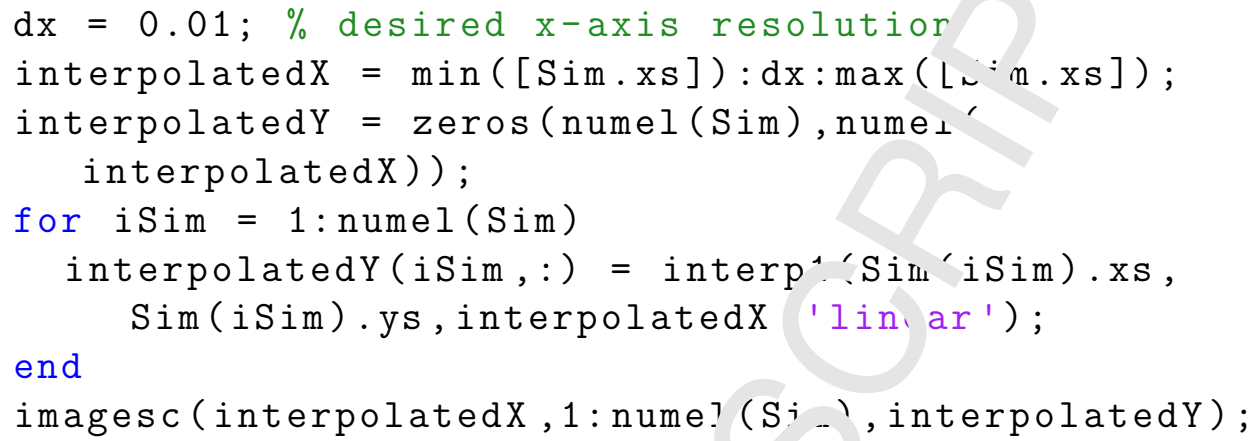

computation time from $\sim 20$ hours to $\sim 3$ minutos. The solution is tailored to sim-

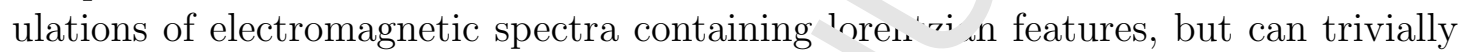
be adapted to other simulation functions and othe. lineshapes. The solution easily plugs into existing simulation systems, and $1_{\llcorner}{ }^{2}$ arfacing to three different simulation systems was demonstrated by examples.

\section{Acknowledgements}

The author gratefully acknowled 5 ......ng from the Danish Council for Strategic Research, DSF, under Grant Agreement No. 10-092322 (PolyNano).

\section{References}

[1] K. Byun, S. Kim, D. Kim, $\Gamma$ sigr stuay of highly sensitive nanowire-enhanced surface plasmon resonance biosensors using iơ $_{0}$ jus $r$ jupled wave analysis., Optics express 13 (10) (2005) 3737 3742. doi:10.1364/OPE'.13.レ`ว 37.

[2] E. Højlund-Nielsen, J. Clau ๆ, T. Mäkela, L. H. Thamdrup, M. Zalkovskij, T. Nielsen, N. Li Pira, J. Ahopelto, N .. Mortensen, A. Kristensen, Plasmonic Colors: Toward Mass Production of Metasurfaces, A ^van ed Materials Technologies 1 (7) (2016) 1600054. doi:10.1002/admt. 201600054.

URL http://d i.wiley.com/10.1002/admt. 201600054

[3] S. A. Boden, D. - B gnall, Optimization of moth-eye antireflection schemes for silicon solar cells, Prog ess in Protovoltaics: Research and Applications 18 (3) (2010) 195-203. doi: 10.1002/i ip. 951.

[4] M. G. wharam, T. K. Gaylord, Rigorous coupled-wave analysis of planar-grating diffraction, Jourr $x$ l of the Optical Society of America 71 (7) (1981) 811. doi:10.1364/JOSA.71.000811. URL L tps: / www .osapublishing. org/abstract.cfm?URI=josa-71-7-811 
[5] M. Shokooh-saremi, R. Magnusson, Particle swarm optimization and its cpplice 'ion to the Design of Diffraction Grating Filters, Optics Letters 32 (8) (2007) 894-89 ${ }^{r}$.

[6] C. Wang, S. Yu, W. Chen, C. Sun, Highly Efficient Light-Trapping Struc • • : Design Inspired By Natural Evolution, Scientific Reports 3 (1) (2013) 1025. doi:10.1し Q/sre 01025.

URL http://www.nature.com/articles/srep01025

[7] B. Rouet-Leduc, K. Barros, E. Cieren, V. Elango, C. Junghans, ז I t s Jkman, J. Mohd-Yusof, R. S. Pavel, A. Y. Rivera, D. Roehm, A. L. McPherson, T. $C$ Ger ${ }_{\Perp}$ nn, Spatial adaptive sampling in multiscale simulation, Computer Physics Comm nicatic s 185 (7) (2014) 18571864. doi:10.1016/j.cpc.2014.03.011.

URL http://dx.doi.org/10.1016/j.cpc.2014.03.011

[8] O. Awile, F. Büyükkeçeci, S. Reboux, I. F. Sbalzarin. ast eighbor lists for adaptiveresolution particle simulations, Computer Physics Com.. 'nic..' .uns 183 (5) (2012) 1073-1081. doi:10.1016/j.cpc.2012.01.003.

URL http://dx.doi.org/10.1016/j.cpc.2012.01. ^n3

[9] K. T. Sørensen, C. B. Ingvorsen, L. H. Nielsen, A. h. 'stensen, Effects of water-absorption and thermal drift on a polymeric photonic cry 7 slab sensor, Optics Express 26 (5) (2018) 5416-5422. doi:10.1364/OE.26.005416.

[10] J. Waxenegger, A. Trügler, U. Hohenester, $\mathrm{P}_{\perp}$ c nonics simulations with the MNPBEM toolbox: Consideration of substrates and layt truct res, Computer Physics Communications 193 (2015) 138-150. arXiv:1412.5467, doi:1c 1 1 . S/j.cpc.2015.03.023.

URL http://dx.doi.org/10.1016/, _,, 1.5 .03 .023$ 